\title{
Experimental Investigations on $\mu$ ED Milling of Inconel 718 with Nano SiC Abrasive Mixed Dielectric
}

\author{
B. Elumalai ${ }^{a *}$ (1), S. Gowri ${ }^{b}$ P. Hariharan ${ }^{b}$, K.V. Arun Pillai \\ ${ }^{a}$ Easwari Engineering College, Department of Mechanical Engineering, Chennai, India. \\ ${ }^{b}$ Anna University, College of Engineering Guindy, Department of Manufacturing Engineering, \\ Chennai, India.
}

Received: September 16, 2021; Revised: October 20, 2021; Accepted: October 31, 2021.

\begin{abstract}
Micro electro-discharge machining is one of the efficient processes to create three-dimensional micro features of metallic components for various applications. Powder mixed EDM improves the machining rate and reduces the surface roughness by evenly distributing the spark. The present studydemonstrates the effect of $\mathrm{SiC}$ nanopowder on the machining of Inconel 718 at different Discharge Energy Regimes (DER). Significant improvement in MRR, reduction in TWR and surface roughness were observed in nanopowder mixed micro-EDM (NPM $\mu \mathrm{EDM})$ compared with micro-EDM. The nano additive considerably improved the Material Removal Rate (MRR) by $163 \%$ and reduced the Tool Wear Rate (TWR)and surface roughness by $24 \%, 17 \%$ respectively. Models were created to predict the Surface Roughness in NPM $\mu$ EDM using two different approaches namely Support Vector Regression (SVR) and Random Forest Machine (RFM). Both SVR and RFM models were able to predict the $\mathrm{R}_{\mathrm{a}}$ value with better accuracies.
\end{abstract}

Keywords: Micro-EDM, Silicon carbide nanoparticle, Inconel 718, Machine learning.

\section{Introduction}

\subsection{Powder mixed EDM}

Electrical discharge machining is one of the nontraditional machining processes adopted by the industries for machining hard conductive materials ${ }^{1-3}$. The control of spark energy becomes a critical factor that affects the material removal process and surface topographywhen the EDM process is used for the creation of micro features. Though the process consumes more time and generates surface imperfections suchas surface cracks, recast layer, and heat-affected zone, researchers are trying to improve the above-mentioned factors by adopting different strategies. The powder mixed EDM process is one of the strategies in which the dielectric liquid is mixed with fine powders. The powders added to the dielectric modify the properties of the dielectric according to the type, size, and concentration and modifying the material removal process dynamics.

Pillai et al. ${ }^{4}$ analyzed the effect of nano-Graphene powder on micro EDM of Ti-6Al-4V alloy with a cryogenic-treated tungsten carbide tool. The results indicate substantial improvements in MRR, TWR, and surface finish due to the cryogenic treatment of the tool and due to the addition of nano-Graphene powder into the dielectric. The obtained results are reasoned with the change in breakdown voltage of dielectric due to the addition of nano-Graphene powder. Paswan et al..$^{5}$ carried out an experimental study on NPMEDM machining of Inconel 718 with nano-Graphene powder mixed dielectric. $20.1 \%, 14 \%$, and $2 \%$ improvement in MRR, surface roughness, and TWR are reported respectively.
They also conducted waveform analysis and found that the spark is more stable in NPMEDM than Conventional EDM.

Abdul-Rani et al. ${ }^{6}$ studied the effect of aluminum nanopowder on NPMEDM of titanium alloy for biomedical applications and observed improvements in surface quality. The reduction in surface cracks and voids was also observed. Chenxue Wang et al. ${ }^{7}$ analyzed NPMEDM with aluminum nanopowder and observed improvements in surface properties. Improvements in MRR and TWR were also observed. Nguyen Huuhan et al. ${ }^{8}$ experimented titanium micro powder mixed EDM process with various tool electrodes. The effect of titanium micro powder and tool material on surface roughness, white layer thickness and hardness were analyzed. The better surface roughness, white layer thickness was obtained when copper electrode used in PMEDM process. The titanium nano powder added to the dielectric significantly increased the hardness of the machined surface.

Hourmand et al. ${ }^{9}$ conducted machining experiments on machining of Al-20Mg2 $\mathrm{Si}$ composite with aluminum nanopowder mixed dielectric and used Adaptiveneuro-fuzzyinferencesystem to model the EDM process. The results of the experiments indicate current as the most significant factor which determines the MRR. The fuzzy model was able to predict the process outcomes effectively. Amorim et al. ${ }^{10}$ studied the effect of zinc coated wire on WEDM of Ti6Al4V alloy. The zinc coating increased the IEG and enabled better flushing improved MRR. The coating also reduced the recast layer thickness considerably. However, no improvement on 
surface quality was reported. Sahu and Mandal ${ }^{11}$ compared the performance of graphite and alumina micro powders in PMEDM of Nimonic 263. Graphite, because of higher electrical and thermal conductivity, produced better MRR whereas alumina produced better surface finish. Current was found as the most significant parameter affecting MRR, TWR and surface finish by a statistical analysis.

Muthuramalingam and Phan ${ }^{12}$ investigated the effect of process parameters on white layer formation in PMEDM process. An attempt was made to correlate the white layer thickness with the aluminum micro powder concentration in the dielectric liquid and other process parameters while machining silicon steel. The results indicated that the aluminum powder in the dielectric reduced the white layer thickness significantly under all operating conditions.

George et al. ${ }^{13}$ investigated the effect of silicon powder with Carbon nano tubes (CNT) and Graphene coated electrodes on EDM of Inconel 825. The results indicated improvements in MRR, TWR and surface finish when CNT coating was used in combination with silicon powder mixed dielectric. Sivaprakasam et al. ${ }^{14}$ experimentally analyzed the effect of graphite nano powder mixed dielectric in micro WEDM process of Inconel 718 and found that the addition of graphite nano powder improved the process characteristics. Improved the topography, surface finish and MRR were reported. Graphite nano powder concentration of $0.5 \mathrm{~g} / 1$, capacitance of $0.01 \mathrm{mF}$ and voltage of $100 \mathrm{~V}$ were found as optimum conditions for better process characteristics.

Talla et al. ${ }^{15}$ also studied the machinability of Inconel 625 with Graphite powder mixed kerosene dielectric. Improvements in surface finish and fatigue strength were reported due to the addition of graphite in the dielectric. Reduction in surface crack density and thickness of the white layer were also reported. Prihandana et al. ${ }^{16}$ examined the impact of molybdenum disulfide $\left(\mathrm{MoS}_{2}\right)$ on EDM machining of Inconel 718. The effect of particle size also investigated. The findings reveal that the $50 \mathrm{~nm} \mathrm{MoS}_{2}$ powder provides better MRR in comparison with $2 \mu \mathrm{m}$ and $10 \mathrm{~nm}$ powders. The study also indicates that the increase in powder concentration under lower energy levels destabilize the process by unwanted secondary discharges and short circuits.

\subsection{Machine Learning in Machining Process}

The complex material removal process in EDM, especially with some additives in the dielectric fluid, makes it difficult to create models. There are various machine learning algorithms successfully used to classify and predict the results in different applications. Different attempts have been made to predict the performance of the machining operations using different models.

Moghaddam and Kolahan ${ }^{17}$ used Artificial Neural Network (ANN) with particle swarm (PSO) algorithm to model EDM process. The proposed back propagation based neural network was able to predict the process outcomes with less than $1 \%$ error. The used optimization technique, particle swarm algorithm, also proved to be effective.Extreme learning machine (ELM) was used to predict surface roughness in abrasive water jet machining process by Ćojbašić et al. ${ }^{18}$. ELM was compared with ANN and genetic algorithms. Better predictive accuracy was obtained with ELM.

Sing Nain et al. ${ }^{19}$ used machine learning algorithms SVM, Geometric Progression (GP) and ANN to predict surface roughness in WEDM process. The predicted values from the different machine learning algorithms were compared. The genetic algorithms provided better prediction accuracy with a small data set. Lu et al. ${ }^{20}$ also compared the prediction accuracies of SVM, GP and PSO algorithms in milling and turning of TC18 titanium alloy. The models were able to predict the surface roughness to appreciable accuracies.

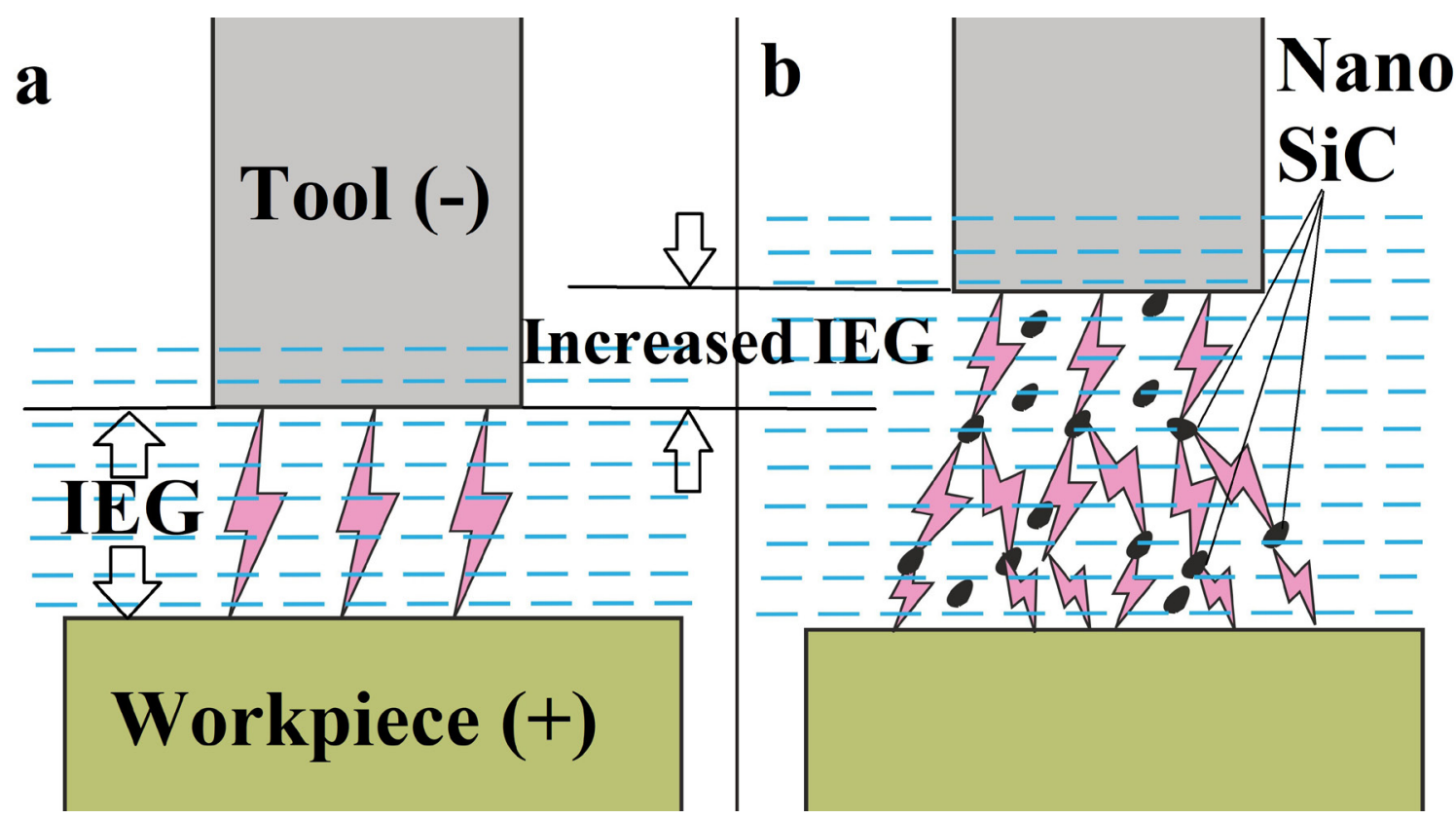

Figure 1. (a) Conventional EDM (b) Nano SiC mixed EDM. 
The SVM algorithm outperformed other mentioned models interms of prediction accuracy and training time.

Ulas et al. ${ }^{21}$ demonstrated application of different machine learning algorithms for prediction of surface roughness in WEDM process. Extreme learning machine and support vector regression were used to build models from the experimental data. Both ELM and SVR models were able to predict surface roughness with high accuracy.

From the literature it is evident that the PMEDM process produces better MRR and surface finish. The addition of powder additives to any dielectric liquid - Distilled water, Kerosene and Hydrocarbon oils, improves the process dynamics. The effect of powder mixed in the dielectric on the performance characteristics of EDM and the mechanism of PMEDM is also studied and analyzed by various researchers. It is also observed that the most of the available literature is on die-sinking EDM process and with expensive conductive powders like Graphene, CNT, and titanium. So there is a need to study effect of powder mixed dielectric in micro-EDM. The effect of nano additives on the white layer formation also needs investigations since very few articles regarding WLT in EDM were found. The present study aims to find the effect of SiC nanoparticle concentration in the dielectric medium while machining Inconel 718 alloy at three different energy levels. MRR, TWR,SR, and WLT were considered as the measurable response parameters.

The detailed literature review also indicates that the data driven machine learning algorithms can be applied to machining processes and can be used to predict one or more output variables. In this study the data from $\mathrm{SiC}$ nanopowder mixed micro ED milling process was used to build SVM and Random Forest Machine (RFM) learning algorithm based models for the prediction of surface roughness.

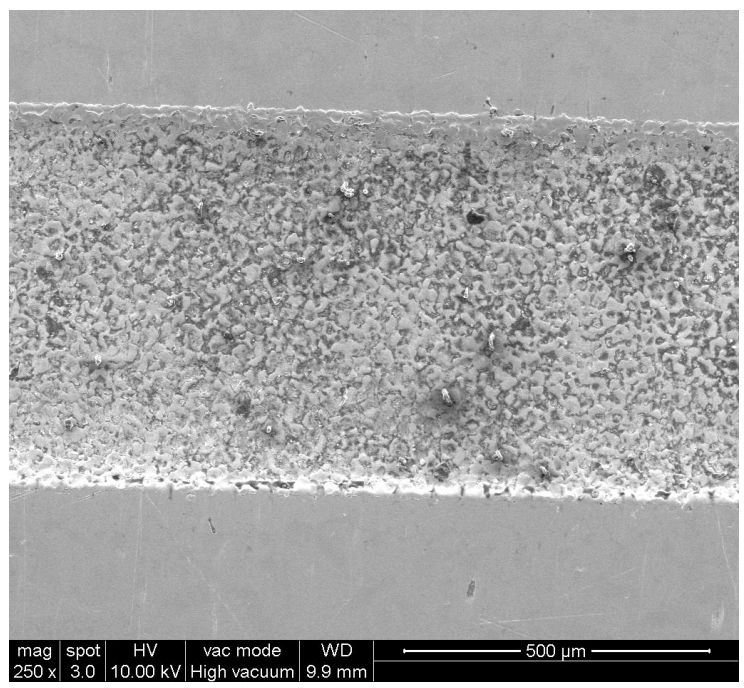

Figure 2. Machined micro slots.

\section{Methodology}

\subsection{Machiningexperiments}

The experiments were conducted in DT-110, a multipurpose micro machining center.Inconel 718 alloy is used as the workpiece. Micro slots, as shown in Figure 2, were machined usinga solid tungsten carbide electrode.Commercially available DCO1000i EDM oil was used as the base dielectric fluid. SiC nanopowder was used as additive with three different concentrations, $0.2 \mathrm{~g} / 1,0.3 \mathrm{~g} / 1$ and $0.4 \mathrm{~g} / 1$, based on literature and previous experiments.

Based on the machine capabilities, three capacitance levels such as $0.1 \mu \mathrm{F}, 0.01 \mu \mathrm{F}$, and $0.001 \mu \mathrm{F}$ (low, medium and high) were considered. The voltage levels such as $80 \mathrm{~V}$, $90 \mathrm{~V}, 100 \mathrm{~V}, 110 \mathrm{~V} \& 120 \mathrm{~V}$ were chosen as the input machine parameters. The full factorial experimental design with sixty experiments was used for this study. The discharge energy $\left[\mathrm{E}=0.5 \mathrm{VC}^{2}\right]$ is classified into three regimes, for analyzing the results, namely low -3.2 to $7.2 \mu \mathrm{J}$, medium -32 to $72 \mu \mathrm{J}$, and high -320 to $720 \mu \mathrm{J}$, as in Table 1 . The workpiece polarity ispositive and the spindle is set to run at a constant speed of $1500 \mathrm{rpm}$.

Before each machining cycle, the tool electrode was flattened at the bottom side by the reverse EDM process. The nano SiC powder (25-35 nm) was measured on an electronic weighing scale and added to the dielectric medium. A magnetic stirrer was used to ensure the consistent mixing of the nanopowder to the dielectric while circulating in the machining chamber. The MRR and TWR were calculated based on the loss of weight after the machining cycle. The surface roughness was measured through a non-contact laser interferometer device. The white layer thickness was measured using a high-resolution SEM.

\subsection{Machine Learning - SVM and RFM}

The surface generated in EDM process is collection of overlapping craters and it depends on various factors. It is difficult to predict the surface roughness in EDM process using analytical equations ${ }^{21}$. The addition of nano $\mathrm{SiC}$ makes the powder mixed micro EDM process more complex to model. Machine learning methods are used to model various processes that are difficult to model using analytical techniques and that are expensive and time consuming ${ }^{17-21}$.

Machine learning isa process of feeding adequate data to train and predict a possible outcome using algorithms. In this study, the Support Vector Machine (SVM) and Random Forest Machine (RFM) techniques are used to build predictive models for predicting surface roughness. The SVM and RFM come under the category of supervised learning, in which the machine is trained with a labeled dataset. The process of building a machine learning model starts with Data acquisition. The data set obtained from full factorial machining experiments was used for training and testing

Table 1. Energy levels classification.

\begin{tabular}{|c|c|c|c|}
\hline Parameter & Unit & & \\
\hline \multirow{3}{*}{ Discharge Energy, $E=\frac{1}{2} C V^{2}$} & \multirow[t]{3}{*}{$\mu \mathrm{J}$} & Low & $3.2,4.05,5,6.05,7.2$ \\
\hline & & Medium & $32,40.5,50,60.5,72$ \\
\hline & & High & $320,405,500,605,720$ \\
\hline
\end{tabular}


the model. The algorithm used for building classification or regression model is based on the nature of the data set. Python was used to create both the SVM and RFM models.

The SVM uses the various kernel functions to convert the inseparable problem into a separable problem by adding more dimensions to it. The generally used kernel functions were linear kernel, polynomial kernel, and radial basis function kernel. The random forest algorithm uses multiple decision trees and it's decided for regression problems.

The obtained data from the full factorial experiments were used to built SVM and Random forest learning algorithms. As the size of the data set is small,5 fold cross validation is used to evaluate the models. Cross-validation is a technique that is used to assess machine learning models on a small sample of data. Cross-validation is largely used in applied machine learning to assess a machine learning model's competence on previously unseen data. That is, to use a small sample to assess how the model will perform in general when used to generate predictions on data that was not utilized during the model's training. It is a popular approach because it is straightforward to comprehend and produces a less biased or optimistic estimate of model competence than other methods. The data set was randomly split into 5 groups. For each unique group: The group was taken as test data set and remaining four groups were used as the training data set. The model was fitted on the test data set to evaluate and the scores ( $\mathrm{R}^{2}$ and RMSE) were recorded. The process was repeated for all five unique groups. The scores were summarized and overall performance of the trained model was evaluated. The models were analyzed based on the Coefficient of Determination $\left(\mathrm{R}^{2}\right)$ and the Root Mean Square Error (RMSE) values.

\section{Results and Discussion}

\subsection{Analysis of $M R R$}

Figure 3 depicts the effect of $\mathrm{SiC}$ nanopowder concentration on MRR under different discharge energy ranges. For all $\mathrm{SiC}$ nanopowder concentrations, under Low and Medium DER, the MRR rises as the discharge energy increases. When the discharge energy is raised, more heat is produced in the machining zone, resulting in an increase in MRR. Under low DER the energy is low and produces weak discharges results in low MRR. Frequent short circuits were observed under low DER. The IEG is very small when the discharge energy is low which causes short circuits. The small IEG also affects the debris removal which results in low MRR.

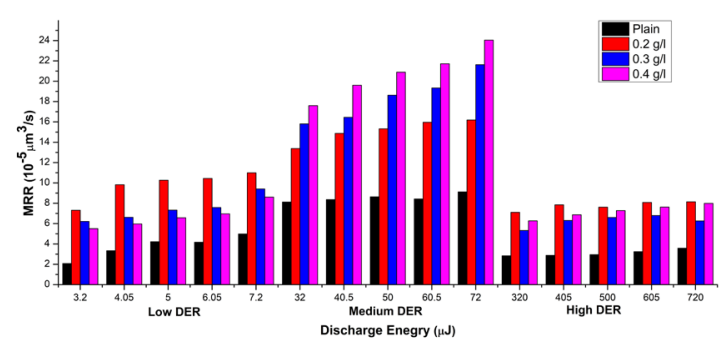

Figure 3. MRR at different Energy Levels Vs SiC concentration.
Under high DER also low MRR is obtained. That can be explained by increased pulse-off time due to high capacitance value. When high capacitance used in an $\mathrm{RC}$ circuit, the charging duration of the capacitor increases that resulted in no spark or pulse-off time. This increase in pulse-off time reduces number of discharges for the given duration. The increase in pulse-off time also enables high convective heat transfer from the workpiece. Thus MRR in high DER is very low when compared with low and high DERs. Better MRR is obtained under medium discharge energy range $(32 \mathrm{uJ}-72 \mathrm{uJ})$ when compared with the low and high DERs. Under medium DER, the combination of sufficient energy for powerful discharges and a short pulse off time improves material removal. Similar results are reported by Pillai et al. ${ }^{4}$.

The material removal rate increased with the increase of $\mathrm{SiC}$ concentration for all discharge energy regimes, as shown in Figure 3. Similar findings were reported by Jahan et al. ${ }^{22}$ and Prakash et al. ${ }^{23}$. The breakdown voltage of the dielectric with various $\mathrm{SiC}$ nanopowder concentrations were measured using a standard breakdown voltage testing equipment with $2 \mathrm{~mm}$ constant IEG. A maximum of $40 \%$ reduction in breakdown voltage was observed with $0.4 \mathrm{~g} / 1$ of $\mathrm{SiC}$ nanopowder concentration. The reduced dielectric breakdown voltage increased the discharge frequency and more sparks were produced for the given time. This resulted in increased MRR. Since the breakdown voltage is reduced due to the addition of $\mathrm{SiC}$ nanopowders, the inter electrode gap is increased. The enlarged IEG makes flushing of debris more efficient, hence improving the material removal process. The larger IEG also allows for a greater number of suspended powder particles in the IEG, creating ideal circumstances for numerous primary and secondary discharges. The $\mathrm{SiC}$ nanoparticles with moderate electrical conductivity also breaks the large discharge channels into smaller and uniformly distributed discharges, as in Figure 1, results in improved erosion. The $\mathrm{SiC}$ being a semiconductor also increased the number intermediate sparks between the tool and the workpiece. The added semi-conductive $\mathrm{SiC}$ has reduced the time to breakdown the dielectric medium and it increased the number of sparks for the given duration. Stable sparking with less frequent arcing was observed when the nano powder mixed dielectric is used ${ }^{5}$. The sparking stability improves as the concentration of $\mathrm{SiC}$ nanopowder increased.

\subsection{Analysis of TWR}

Figure 4 portrays the TWR as function of discharge energy and $\mathrm{SiC}$ nanopowder concentration. The TWR increases with the increase in discharge energy under low and medium DER. As the discharge energy increases, the heat generation in the machining zone increases. The increase in heat enables more material removal from both workpiece and tool electrodes. Significant reduction in TWR was observed under high DER. The high DER is obtained with high capacitance value and thus increases the charging duration of the $\mathrm{RC}$ circuit $^{24}$. This charging duration in $\mathrm{RC}$ circuit can be considered equivalent to pulse-off time in conventional EDM circuit. Thus the increase in pulse-off time resulted in reduced material removal both from workpiece and tool. Under high DER the plasma channel widens and the spark expands because of high discharge duration and energy level. 
As a result, the bulk of the plasma's heat is transferred to the dielectric and workpiece, reducing heat transfer to the too ${ }^{11}$ which also reduces TWR under high DER.

The addition of $\mathrm{SiC}$ nanopowder enables better sparking in low DER. The increase in sparking frequency as the result of reduced breakdown voltage of the dielectric increases the material removal in workpiece and tool. Thus the addition of SiC nanoparticles under low DER increases the TWR. The increase in $\mathrm{SiC}$ nanopowder concentration under low DER increases the TWR significantly and it is depicted in Figure 4. Under medium and high DER, the addition of

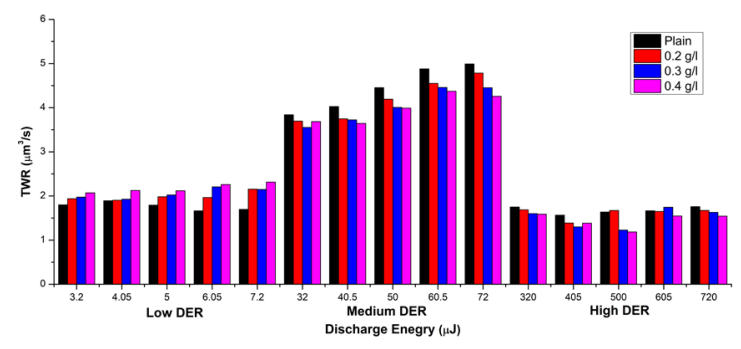

Figure 4. Tool Wear Rate Vs SiC at different Energy Levels.

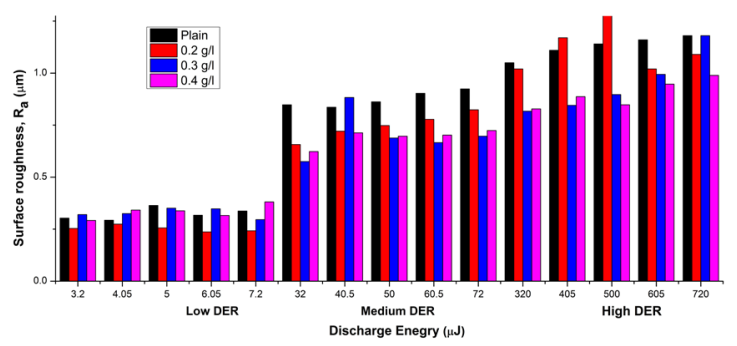

Figure 5. Surface roughness $\mathrm{Vs} \mathrm{SiC}$ and Energy Level.
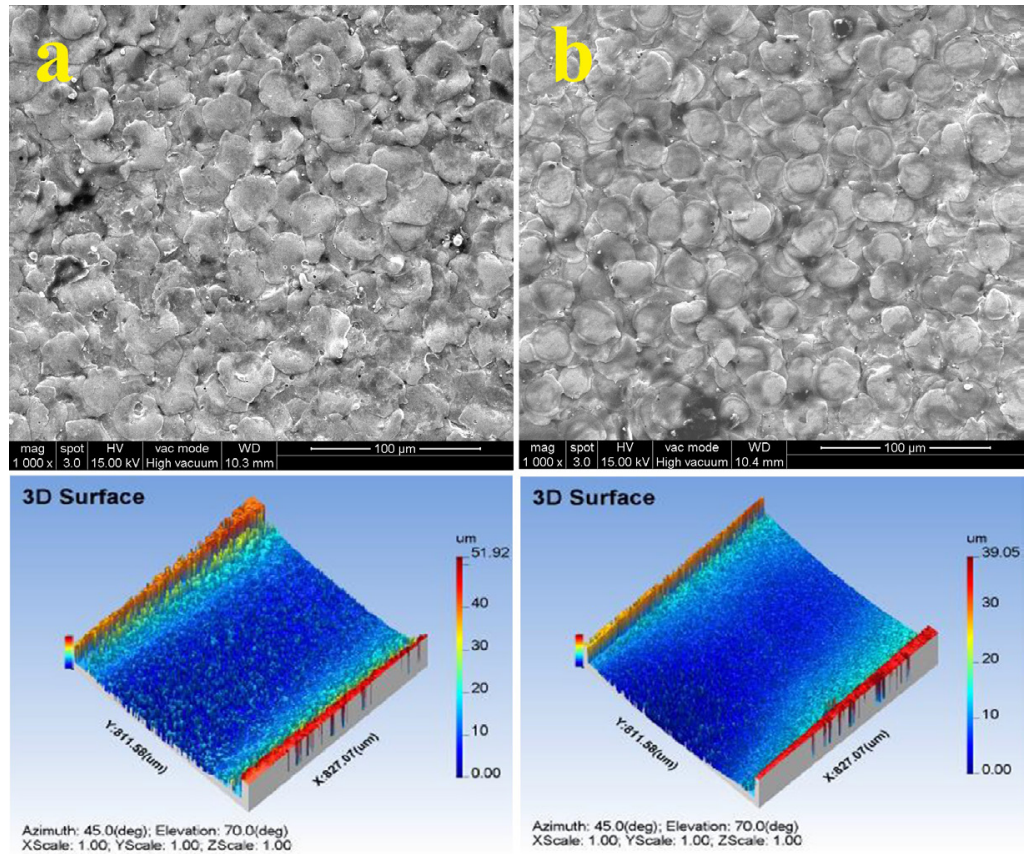

Figure 6. SEM and 3D surface images of the level of energy applied during the machined surface (a) Plain dielectric and (b)SiC mixed.
$\mathrm{SiC}$ nanopowder reduced the TWR considerably. The TWR is the result of melting and evaporation of tool electrode due the heat generated during discharges. Due to the high heat generated under medium and high DER pyrolysis of hydrocarbon based dielectric takes place. The carbon from the pyrolysis process deposited on the tool electrode reduces the thermal conductivity of the tungsten carbide electrode and results in increased TWR ${ }^{25}, 26$. The SiC nanopowder breaks the large plasma channels into smaller and less intense channels. This reduces the pyrolysis rate and hence reduction in carbon deposition. The $\mathrm{SiC}$ nanoparticels also reduces the carbon deposition on the tool surface by frequently striking it. The reduction in carbide layer due to the $\mathrm{SiC}$ nanopowder resulted in reduced TWR under medium and high DER. The SiC nanoparticles also collide with the proton discharge, ions and electrons from the anode ${ }^{27}$ and reduced the amount of erosion at the cathode (tool). The increase in $\mathrm{SiC}$ concentration reduces the TWR in medium and high DER as depicted in Figure 4.

\subsection{Analysis of surface finish}

Figure 5 represents the Ra value of the machined surface with respect to the nanopowder concentration and discharge energy. The surface roughness increases with the increase in discharge energy. The increase in discharge energy increases the surface roughness under all three DERs. The increase in discharge energy increases the spark intensity and size. The increased spark size results in increased crater size. The surface generated in EDM process is the result of multiple overlapping craters as shown in Figure 6. Thus the increase in discharge energy increases the crater size and hence increases the surface roughness.

The addition of $\mathrm{SiC}$ nanopowder in all DERs resulted in reduced surface roughness. The addition of nanopowder reduces the breakdown voltage and increase the IEG. The SiC

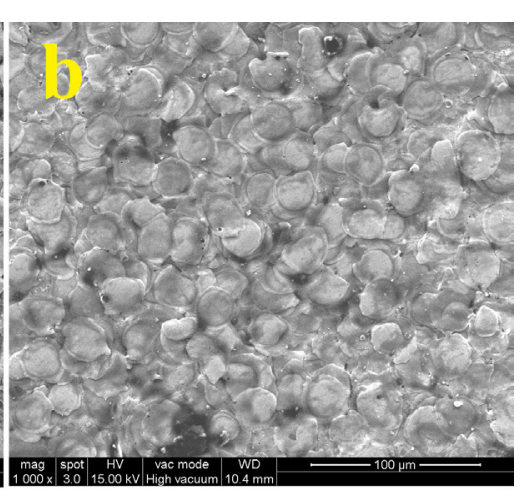




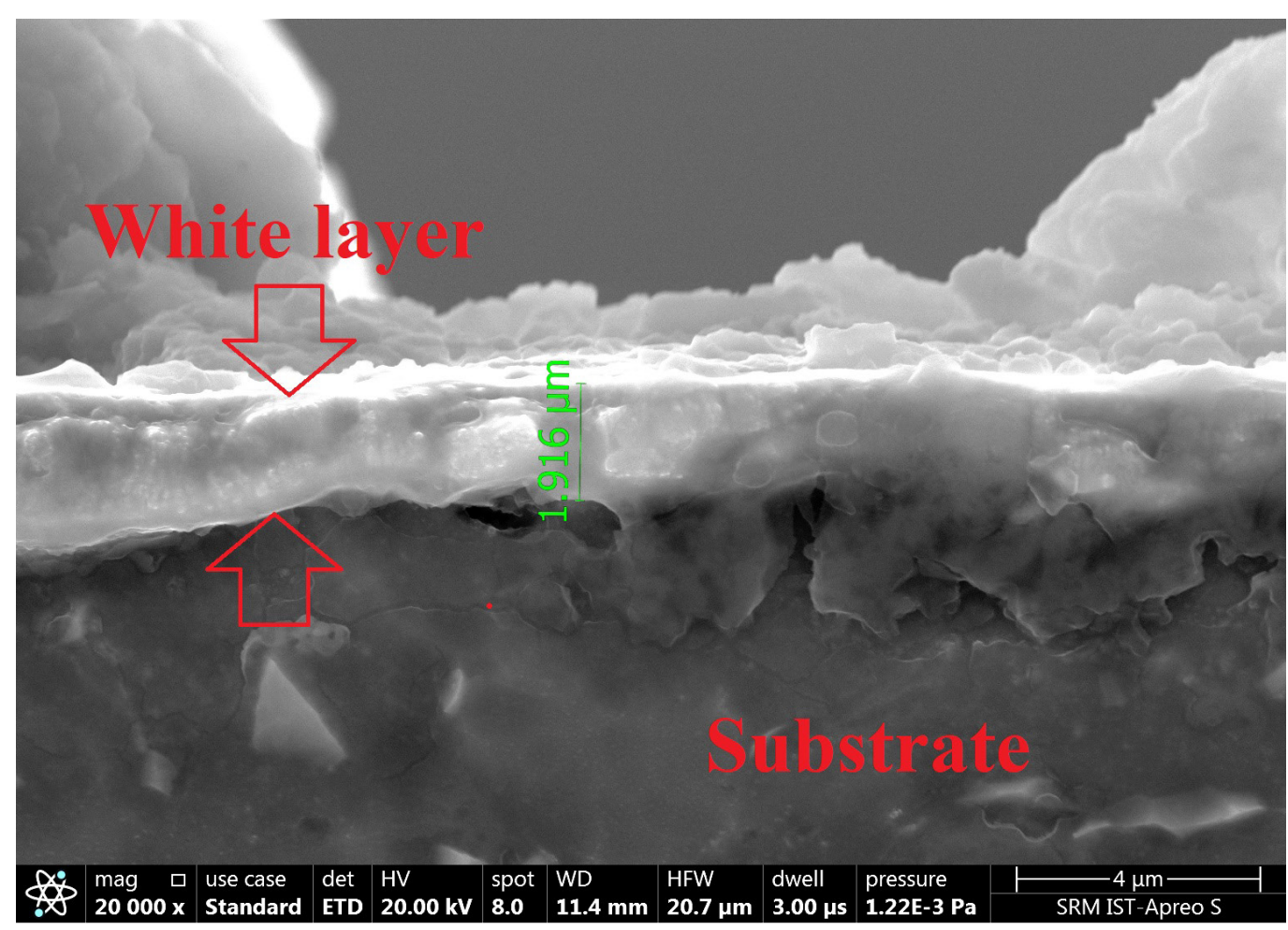

Figure 7. White layer.

nanopowder breaks large discharge channels and enable uniformly distributed smaller sparks with less intensity. The reduced per spark intensity reduces the crater size and reduced the surface roughness ${ }^{28,29}$. The SEM and 3D surface images of the machined surfaces with and without nano $\mathrm{SiC}$ addition are shown in Figure 7. Small shallow craters with rim were observed when the nano $\mathrm{SiC}$ is mixed with the dielectric, (Figure 6b) whereas larger, uneven craters without any rim like feature observed with pure dielectric (Figure 6a). The SiC abrasive nanopowder may also reduced the surface roughness due abrasive action over the machined surface as the particles moves with high velocity due to plasma burst and also due to the rotation of tool electrode. Similar findings were reported by Baghel et al. ${ }^{30} \mathrm{Wu}$ et al. ${ }^{31}$.

\subsection{White layer thickness}

The white layer thickness values were measured for the slots machined at the voltage of $120 \mathrm{~V}$. From the measured values of the recast layer, it is evident that both Energy level/capacitance and the quantity of nano $\mathrm{SiC}$ added to the dielectric affect the recast layer thickness. Figure 7 represents the white layer thickness for various concentrations of $\mathrm{SiC}$ nanopowder at different energy levels. A distinct amorphous layer of various thicknesses is found at the top surface of all the microchannels machined (Figure8).

The white layer thickness, when the pure dielectric oil is used, is proportional to the discharge energy and follows the semi-empirical model of Lee et al. ${ }^{32}$. The addition of nano $\mathrm{SiC}$ reduced the WLT at all energy levels. As the number of sparks increased due to the presence of the nano $\mathrm{SiC}$ additives, the discharge energy per spark gets reduced and

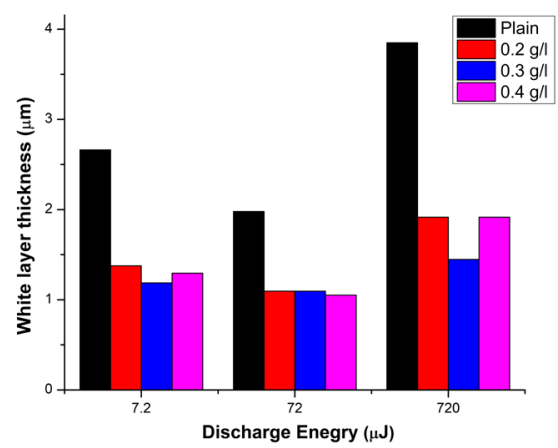

Figure 8. Effect of SiC concentration and discharge energy on WLT.

it resulted in reduced recast and heat affected zone; hence the reduced WLT. The suspended additive particles remove the molten layer from the machining zone and further reduce the formation of thick white layer ${ }^{33}$.

\subsection{Machine learning}

Four types of kernel functions such as linear, polynomial, sigmoid, and radial basis functions were used to create the roughness prediction model. It was transmitted to the regression analysis which has a measure of the relation between the mean value of one variable and the corresponding value of other variables. Among the four kernel functions used in SVR the Polynomial kernel with order 3 and RBF kernel created better models. The experimental data and the predicted values of surface roughness by SVR and Random forest machines are presented in Table 2. The $\mathrm{R}$ squared and 
Table 2. Experimental and Predicted Surface Roughness for various.

\begin{tabular}{|c|c|c|c|c|c|c|c|c|}
\hline \multirow{3}{*}{ S1. No. } & \multirow{3}{*}{$\begin{array}{c}\text { Capacitance } \\
(\mu \mathrm{F})\end{array}$} & \multirow{3}{*}{ Voltage (V) } & \multirow{3}{*}{ Energy $(\mu \mathrm{J})$} & \multirow{3}{*}{$\begin{array}{c}\text { Powder } \\
(\mathrm{g} / \mathrm{L})\end{array}$} & \multicolumn{4}{|c|}{$\mathrm{Ra}(\mu \mathrm{m})$} \\
\hline & & & & & \multirow[b]{2}{*}{ Actual } & \multicolumn{3}{|c|}{ Predicted } \\
\hline & & & & & & $\begin{array}{c}\text { SVR } \\
\text { (Polynomial) }\end{array}$ & SVR (RBF) & $\begin{array}{c}\text { Random } \\
\text { Forest }\end{array}$ \\
\hline 1 & 0.001 & 80 & 3.2 & 0 & 0.303 & 0.413 & 0.433 & 0.435 \\
\hline 2 & 0.001 & 90 & 4.05 & 0 & 0.293 & 0.298 & 0.294 & 0.338 \\
\hline 3 & 0.001 & 100 & 5 & 0 & 0.364 & 0.362 & 0.366 & 0.396 \\
\hline 4 & 0.001 & 110 & 6.05 & 0 & 0.317 & 0.314 & 0.319 & 0.369 \\
\hline 5 & 0.001 & 120 & 7.2 & 0 & 0.337 & 0.340 & 0.336 & 0.357 \\
\hline 6 & 0.01 & 80 & 32 & 0 & 0.848 & 0.713 & 0.692 & 0.732 \\
\hline 7 & 0.01 & 90 & 40.5 & 0 & 0.836 & 0.839 & 0.837 & 0.841 \\
\hline 8 & 0.01 & 100 & 50 & 0 & 0.862 & 0.860 & 0.863 & 0.843 \\
\hline 9 & 0.01 & 110 & 60.5 & 0 & 0.903 & 0.907 & 0.905 & 0.841 \\
\hline 10 & 0.01 & 120 & 72 & 0 & 0.924 & 0.921 & 0.926 & 0.875 \\
\hline 11 & 0.1 & 80 & 320 & 0 & 1.05 & 1.054 & 1.049 & 0.969 \\
\hline 12 & 0.1 & 90 & 405 & 0 & 1.11 & 1.107 & 1.110 & 1.038 \\
\hline 13 & 0.1 & 100 & 500 & 0 & 1.14 & 1.141 & 1.139 & 1.110 \\
\hline 14 & 0.1 & 110 & 605 & 0 & 1.16 & 1.029 & 0.976 & 0.981 \\
\hline 15 & 0.1 & 120 & 720 & 0 & 1.18 & 1.177 & 1.180 & 1.108 \\
\hline 16 & 0.001 & 80 & 3.2 & 0.2 & 0.253 & 0.259 & 0.254 & 0.361 \\
\hline 17 & 0.001 & 90 & 4.05 & 0.2 & 0.274 & 0.276 & 0.279 & 0.309 \\
\hline 18 & 0.001 & 100 & 5 & 0.2 & 0.256 & 0.265 & 0.259 & 0.337 \\
\hline 19 & 0.001 & 110 & 6.05 & 0.2 & 0.237 & 0.241 & 0.241 & 0.303 \\
\hline 20 & 0.001 & 120 & 7.2 & 0.2 & 0.242 & 0.255 & 0.245 & 0.311 \\
\hline 21 & 0.01 & 80 & 32 & 0.2 & 0.657 & 0.669 & 0.660 & 0.655 \\
\hline 22 & 0.01 & 90 & 40.5 & 0.2 & 0.721 & 0.736 & 0.722 & 0.775 \\
\hline 23 & 0.01 & 100 & 50 & 0.2 & 0.748 & 0.758 & 0.750 & 0.757 \\
\hline 24 & 0.01 & 110 & 60.5 & 0.2 & 0.778 & 0.784 & 0.775 & 0.759 \\
\hline 25 & 0.01 & 120 & 72 & 0.2 & 0.824 & 0.816 & 0.824 & 0.799 \\
\hline 26 & 0.1 & 80 & 320 & 0.2 & 1.02 & 1.012 & 1.016 & 0.941 \\
\hline 27 & 0.1 & 90 & 405 & 0.2 & 1.17 & 1.157 & 1.166 & 1.005 \\
\hline 28 & 0.1 & 100 & 500 & 0.2 & 1.43 & 1.415 & 1.350 & 1.112 \\
\hline 29 & 0.1 & 110 & 605 & 0.2 & 1.02 & 1.019 & 1.015 & 0.977 \\
\hline 30 & 0.1 & 120 & 720 & 0.2 & 1.09 & 1.087 & 1.087 & 1.101 \\
\hline 31 & 0.001 & 80 & 3.2 & 0.3 & 0.32 & 0.315 & 0.321 & 0.364 \\
\hline 32 & 0.001 & 90 & 4.05 & 0.3 & 0.325 & 0.319 & 0.329 & 0.310 \\
\hline 33 & 0.001 & 100 & 5 & 0.3 & 0.351 & 0.337 & 0.349 & 0.349 \\
\hline 34 & 0.001 & 110 & 6.05 & 0.3 & 0.348 & 0.332 & 0.346 & 0.313 \\
\hline 35 & 0.001 & 120 & 7.2 & 0.3 & 0.296 & 0.302 & 0.297 & 0.325 \\
\hline 36 & 0.01 & 80 & 32 & 0.3 & 0.575 & 0.591 & 0.578 & 0.622 \\
\hline 37 & 0.01 & 90 & 40.5 & 0.3 & 0.883 & 0.697 & 0.704 & 0.716 \\
\hline 38 & 0.01 & 100 & 50 & 0.3 & 0.688 & 0.699 & 0.691 & 0.717 \\
\hline 39 & 0.01 & 110 & 60.5 & 0.3 & 0.666 & 0.668 & 0.669 & 0.708 \\
\hline 40 & 0.01 & 120 & 72 & 0.3 & 0.697 & 0.704 & 0.702 & 0.733 \\
\hline 41 & 0.1 & 80 & 320 & 0.3 & 0.817 & 0.822 & 0.820 & 0.861 \\
\hline 42 & 0.1 & 90 & 405 & 0.3 & 0.845 & 0.849 & 0.846 & 0.940 \\
\hline 43 & 0.1 & 100 & 500 & 0.3 & 0.897 & 0.898 & 0.900 & 1.018 \\
\hline 44 & 0.1 & 110 & 605 & 0.3 & 0.994 & 0.986 & 0.990 & 0.930 \\
\hline 45 & $\begin{array}{l}0.1 \\
\end{array}$ & 120 & 720 & 0.3 & 1.18 & 1.165 & 1.175 & 1.091 \\
\hline 46 & 0.001 & 80 & 3.2 & 0.4 & 0.292 & 0.308 & 0.381 & 0.383 \\
\hline 47 & 0.001 & 90 & 4.05 & 0.4 & 0.342 & 0.336 & 0.340 & 0.326 \\
\hline 48 & 0.001 & 100 & 5 & 0.4 & 0.338 & 0.323 & 0.342 & 0.359 \\
\hline 49 & 0.001 & 110 & 6.05 & 0.4 & 0.316 & 0.305 & 0.320 & 0.316 \\
\hline 50 & 0.001 & 120 & 7.2 & 0.4 & 0.381 & 0.374 & 0.375 & 0.333 \\
\hline 51 & 0.01 & 80 & 32 & 0.4 & 0.623 & 0.613 & 0.620 & 0.636 \\
\hline 52 & 0.01 & 90 & 40.5 & 0.4 & 0.713 & 0.707 & 0.707 & 0.728 \\
\hline 53 & 0.01 & 100 & 50 & 0.4 & 0.697 & 0.704 & 0.698 & 0.728 \\
\hline 54 & 0.01 & 110 & 60.5 & 0.4 & 0.702 & 0.715 & 0.706 & 0.724 \\
\hline 55 & 0.01 & 120 & 72 & 0.4 & 0.724 & 0.740 & 0.751 & 0.755 \\
\hline 56 & 0.1 & 80 & 320 & 0.4 & 0.828 & 0.838 & 0.830 & 0.875 \\
\hline 57 & 0.1 & 90 & 405 & 0.4 & 0.887 & 0.894 & 0.887 & 0.945 \\
\hline 58 & 0.1 & 100 & 500 & 0.4 & 0.848 & 0.852 & 0.848 & 1.024 \\
\hline 59 & 0.1 & 110 & 605 & 0.4 & 0.947 & 0.944 & 0.943 & 0.937 \\
\hline 60 & 0.1 & 120 & 720 & 0.4 & 0.989 & 1.000 & 0.990 & 1.076 \\
\hline
\end{tabular}




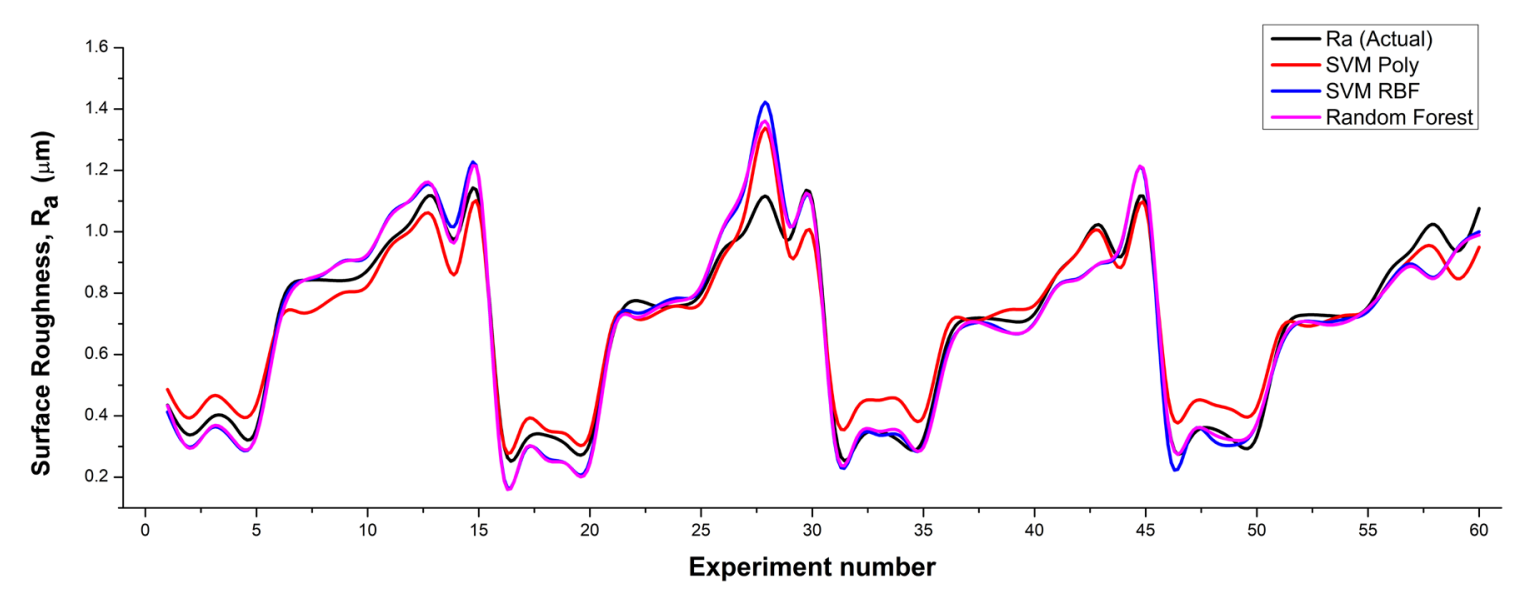

Figure 9. Actual Vs predicted roughness.

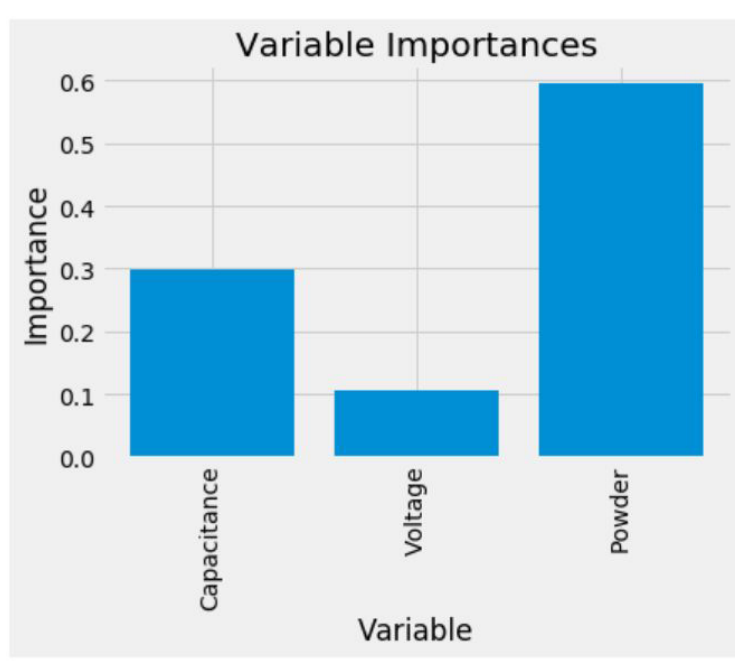

Figure 10. Variable importance matrix from Random Forest Machine.

Table 3. Performance of Machine learning models.

\begin{tabular}{lcc}
\hline \multicolumn{1}{c}{ Model } & $\mathbf{R}^{\mathbf{2}}$ & RMSE \\
\hline Polynomial-SVR & 0.979 & 0.045 \\
\hline RBF-SVR & 0.986 & 0.038 \\
\hline Random Forest machine & 0.934 & 0.081 \\
\hline
\end{tabular}

RMSE values for each kernel are also presented in Table 3. Figure 9 displays the actual and model-predicted values of surface roughness for polynomial-SVR, RBF-SVR and Random forest machine. Figure 10 depicts the importance of the three input variables for deciding the surface roughness according to the random forest machine.

\section{Conclusions}

In this work the influence of $\mathrm{SiC}$ nanopowder and its concentration on nano powder mixed micro electro discharge milling of Inconel 718 under three different energy regimes were investigated. Material removal rate, tool wear rate and surface roughness were considered as performance measures for this investigation. Machine learning algorithms were used to build prediction models from the experimental data. The following conclusions can be drawn from the experimental results.

- $\quad$ The medium DER produced better MRR due to optimum charging and discharging duration and increased erosion efficiency.

- An average of $163.58 \%$ improvement in MRR was observed with $0.4 \mathrm{~g} / 1$ of $\mathrm{SiC}$ nanopowder concentration under medium DER.

- The addition of SiC nanopowder reduced the TWR up to $24.35 \%$ under medium and high DERs.

- An average reduction of $17.20 \%$ in surface roughness was obtained with $0.4 \mathrm{~g} / 1$ of $\mathrm{SiC}$ nanopowder concentration.

- The addition of SiCnanopowderi in the dielectric reduced the WLT considerably.

- $\quad$ SVR with polynomial and RBF kernals predicted the surface roughness in NPM $\mu \mathrm{EDM}$ with $\mathrm{R}^{2}$ values of 0.979 and 0.986 respectively.

\section{References}

1. Gupta V, Singh B, Mishra RK. Machining of titanium and titanium alloys by electric discharge machining process: a review. Int J Mach Mach Mater. 2020;22(2):99-121.

2. Quarto M, Bissacco G, D’Urso G. Machinability and energy efficiency in micro-EDM milling of zirconium boride reinforced with silicon carbide fibers. Materials. 2019;12(23):3920.

3. Carlini GC, Moura CR, Amorim FL, Weingaertner WL. On geometrical characteristics of WC-Co round cavities after ED-machining with different grades of $\mathrm{CuW}$ electrodes. Mater Res. 2020;23(2):e20200041.

4. Pillai KVA, Hariharan P, Jafferson JM. $\mu$ ED milling of Ti$6 \mathrm{Al}-4 \mathrm{~V}$ using cryogenic-treated $\mathrm{Wc}$ tool and nano-graphene powder-mixed dielectricat different discharge energy regimes. Int J Adv Manuf Technol. 2019;102(9-12):2721-43.

5. Paswan K, Pramanik A, Chattopadhyaya S. Machining performance of Inconel 718 using graphene nanofluid in EDM. Mater Manuf Process. 2020;35(1):33-42. 
6. Abdul-Rani AM, Nanimina AM, Ginta TL, Razak MA. Machined surface quality in nano aluminum mixed electrical discharge machining. Procedia Manuf. 2017;7:510-7.

7. Wang C, Qiang Z. Comparison of micro-EDM characteristics of inconel 706 between EDM Oil and an al powder-mixed dielectric. Adv Mater Sci Eng. 2019;2019:5625360.

8. HuuPhan N, Muthuramalingam T, Vu NN, Tuan NQ. Influence of micro size titanium powder-mixed dielectric medium on surface quality measures in EDM process. Int J Adv Manuf Technol. 2020;109(3-4):797-807.

9. Hourmand M, Sarhan AAD, Farahany S, Sayuti M. Microstructure characterization and maximization of the material removal rate in nano-powder mixed EDM of Al-Mg 2 Si metal matrix composite: ANFIS and RSM approaches. Int J Adv Manuf Technol. 2019;101(9-12):2723-37.

10. Amorim FL, Torres RD, Laurindo CAH, Reolon LW. Performance and surface integrity of wire electrical discharge machining of thin Ti6Al4V plate using coated and uncoated wires. Mater Res. 2019;22(3):4-9.

11. Sahu DR, Mandal A. Critical analysis of surface integrity parameters and dimensional accuracy in powder-mixed EDM. Mater Manuf Process. 2020;35(4):430-41.

12. Muthuramalingam T, Phan NH. Experimental Investigation of white layer formation on machining silicon steel in PMEDM process. Silicon. 2021;13(7):2257-63.

13. George J, Chandan R, Manu R, Mathew J. Experimental investigation of silicon powder mixed EDM using graphene and CNT nano particle coated electrodes. Silicon. 2020;13:3835-51.

14. Sivaprakasam $P$, Hariharan P, Gowri S. Experimental investigations on nano powder mixed Micro-Wire EDM process of inconel-718 alloy. Meas J Int Meas Confed. 2019;147:106844.

15. Talla G, Gangopadhyay S, Biswas CK. Influence of graphite powder mixed EDM on the surface integrity characteristics of Inconel 625. Particul Sci Technol. 2017;35(2):219-26.

16. Prihandana GS, Sriani T, Mahardika M, Hamdi M, Miki N, Wong YS, et al. Application of powder suspended in dielectric fluid for fine finish micro-EDM of Inconel 718. Int J Adv Manuf Technol. 2014;75(1-4):599-613.

17. Moghaddam MA, Kolahan F. Modeling and optimization of the electrical discharge machining process based on a combined artificial neural network and particle swarm optimization algorithm. Sci Iran. 2020;27(3):1206-17.

18. Ćojbašić Ž, Petković D, Shamshirband S, Tong CW, Ch S, Janković $P$, et al. Surface roughness prediction by extreme learning machine constructed with abrasive water jet. Precis Eng. 2016;43:86-92.

19. Singh Nain S, Sai R, Sihag P, Vambol S, Vambol V. Use of machine learning algorithm for the better prediction of SR peculiarities of WEDM of Nimonic-90 superalloy. Arch Mater Sci Eng. 2019;95(1):12-9.
20. Lu J, Liao X, Li S, Ouyang H, Chen K, Huang B. An effective ABC-SVM approach for surface roughness prediction in manufacturing processes. Complexity. 2019;2019:3094670.

21. Ulas M, Aydur O, Gurgenc T, Ozel C. Surface roughness prediction of machined aluminum alloy with wire electrical discharge machining by different machine learning algorithms. J Mater Res Technol. 2020;9(6):12512-24.

22. Jahan MP, Rahman M, Wong YS. Modelling and experimental investigation on the effect of nanopowder-mixed dielectric in micro-electrodischarge machining of tungsten carbide. Proc Inst Mech Eng, B J Eng Manuf. 2010;224(11):1725-39.

23. Prakash C, Kansal HK, Pabla BS, Puri S. Experimental investigations in powder mixed electric discharge machining of Ti-35Nb-7Ta-5Zr $\beta$-titanium alloy. Mater Manuf Process. 2017;32(3):274-85.

24. Rani SS, Sundari VK, Jose PSH, Sivaranjani S, Stalin B, Pritima D. Enrichment of material subtraction rate on Eglin steel using electrical discharge machining process through modification of electrical circuits. Mater Today Proc. 2020;33(7):4428-30.

25. Sahu SK, Datta S. Experimental studies on graphite powdermixed electro-discharge machining of Inconel 718 super alloys: comparison with conventional electro-discharge machining. Proc Inst Mech Eng, E J Process Mech Eng. 2019;233(2):384-402.

26. Perumal A, Kailasanathan C, Stalin B, Rajkumar PR, Gangadharan T, Venkatesan G. Evaluation of EDM process parameters on titanium alloy through Taguchi approach. Mater Today Proc. 2021;45(2):2394-400.

27. Li L, Zhao L, Li ZY, Feng L, Bai X. Surface characteristics of Ti-6Al-4V by SiC abrasive mixed EDM with magnetic stirring. Mater Manuf Process. 2017;32(1):83-6.

28. Zhu Z, Guo D, Xu J, Lin J, Lei J, Xu B, et al. Processing characteristics of micro electrical discharge machining for surface modification of TiNi shape memory alloys using a TiC powder dielectric. Micromachines. 2020;11(11):1-15.

29. Mughal MP, Farooq MU, Mumtaz J, Mia M, Shareef M, Javed $\mathrm{M}$, et al. Surface modification for osseointegration of Ti6Al4V ELI using powder mixed sinking EDM. J Mech Behav Biomed Mater. 2021;113:104145.

30. Baghel R, Biswas SK. Micro hole fabrication in $\mathrm{TiN}-\mathrm{Al}_{2} \mathrm{O}_{3}$ ceramic composite by $\mathrm{SiC}$ powder assisted micro- EDM. Engineering Research Express. 2020;2(1):15028.

31. Wu Z, Luo F, Guo D, Wu X, Xu B, Lei J, et al. Micro-EDM by using laminated $3 \mathrm{D}$ microelectrodes with deionized water containing $\mathrm{B}_{4} \mathrm{C}$ powder. Int J Adv Manuf Technol. 2018;99:2893902.

32. Lee LC, Lim LC, Narayanan V, Venkatesh VC. Quantification of surface damage of tool steels after EDM. Int J Mach Tools Manuf. 1988;28(4):359-72.

33. Kibria G, Sarkar BR, Pradhan BB, Bhattacharyya B. Comparative study of different dielectrics for micro-EDM performance during microhole machining of Ti-6Al-4V alloy. Int J Adv Manuf Technol. 2010;48(5-8):557-70. 